\title{
Driveless Gyroscope Response of a Quartz Piezoelectric Vibratory Tuning Fork
}

\author{
M. R. Abdel-Rahman ${ }^{a, *}$, B. A. Albassam ${ }^{b}$, M. A. Alduraibi ${ }^{c, d}$ \\ ${ }^{a}$ Prince Sultan Advanced Technologies Research Institute (PSATRI), College of Engineering, \\ King Saud University, Riyadh 11421, Saudi Arabia \\ ${ }^{b}$ Mechanical Engineering Department, College of Engineering, King Saud University, Riyadh 11421, Saudi Arabia \\ ${ }^{c}$ Physics and Astronomy Department, College of Science, King Saud University, Riyadh 11451, Saudi Arabia \\ ${ }^{d}$ National Center for Applied Physics, King Abdulaziz City for Science and Technology, KACST, P.O. Box 6086, \\ Riyadh 11442, Saudi Arabia
}

\begin{abstract}
In this paper, we report on the observation of a gyroscope response in the absence of drive oscillator circuitry in a single ended quartz piezoelectric tuning fork. The tuning fork gyroscope was fabricated by conventional photolithography and wet etching techniques. The tuning fork length is $4 \mathrm{~mm}$ and the stem length is $2 \mathrm{~mm}$; the tuning fork width and thickness are $0.4 \mathrm{~mm}$ and $0.35 \mathrm{~mm}$, respectively. The measured gyroscope sensitivity and signal to noise ratio were $0.22 \mathrm{mV} / \% / \mathrm{s}$ and 40 , respectively, at a rotational rate of $60 \% / \mathrm{s}$.
\end{abstract}

DOI: 10.12693/APhysPolA.127.1352

PACS: $85.85 .+\mathrm{j}$

\section{Introduction}

The studies of the quartz tuning fork gyroscopes have been used in a variety of commercial and military applications, as they have proven to be highly sensitive, highly accurate and highly stable against temperature and time. In addition, they have shown high rigidity and they are easy to fabricate [1-2]. A quartz tuning fork gyroscope operates in such a way that a drive oscillator circuit is used to excite the tuning fork tines in the drive mode with a certain frequency. This excitation signal will result in the tuning fork tines moving opposite to each other in the tuning fork plane. If the tuning fork rotates at a certain angular rotational rate, then a torque, which is proportional to the rotational rate, will be produced by virtue of Coriolis effect. The tines will respond to this torque by moving opposite to each other out of the tuning fork plane. Due to the piezoelectric nature of the material composing the sensor, this motion will produce an electric signal that is proportional to the rotational rate [3-4]. In this work, we fabricate a single ended piezoelectric tuning fork and we report on the observation of a gyroscope response with a single tine of the quartz tuning fork in the absence of a drive oscillator.

\section{Device structure and simulation}

The sensor's geometrical structure and sense electrode positioning are shown in Fig. 1. The tuning fork length is $4 \mathrm{~mm}$ and the stem length is $2 \mathrm{~mm}$; the tuning fork

${ }^{*}$ corresponding author; e-mail: mabdelrahman@ksu.edu.sa width and thickness are $0.4 \mathrm{~mm}$ and $0.35 \mathrm{~mm}$, respectively. The implemented sense electrode positioning on the top, outer and inner faces of the tuning fork is shown in Fig. 1a, Fig. 1b and Fig. 1c respectively. The structure was simulated using COVENTOR software. The simulated drive and sense modes of vibration are shown in Fig. 2. If the tuning fork is rotated with a certain angular velocity, the tines will tend to move away from the axis of rotation due to centrifugal forces. This motion type is characterized as the drive mode, as shown in Fig. 2a. Due to angular rotation, the tines will simultaneously be exposed to Coriolis forces which will move the tines in a direction perpendicular to the drive mode. The resulting motion type is called the sense mode and it is shown in Fig. 2b.

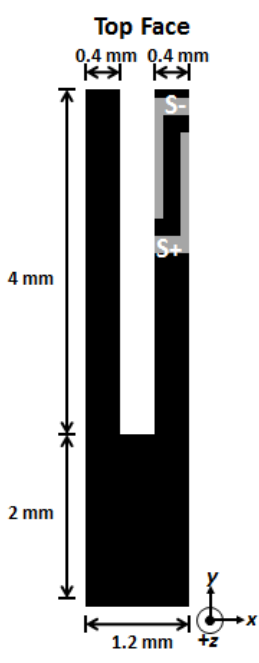

(a)

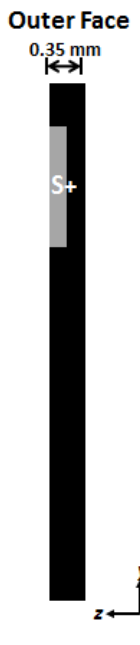

(b)

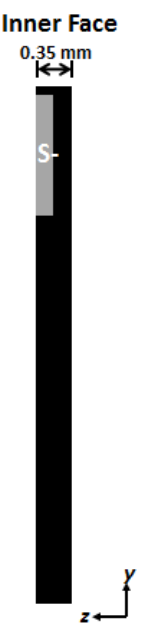

(c)
Fig. 1. Two-dimensional schematic for the tuning fork structure: a) top face, b) outer face, and c) inner face. 


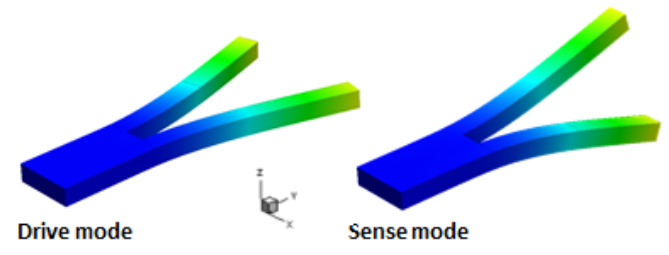

(a)

(b)

Fig. 2. Simulated drive and sense modes of vibration: a) drive mode, and b) sense mode.

\section{Device fabrication}

Double side polished $z$-cut optical grade quartz wafers were used in this work. After cleaning, the wafers were sputter coated with chromium $(\mathrm{Cr})$ and gold $(\mathrm{Au}) ; 20 \mathrm{~nm}$ of $\mathrm{Cr}$ followed by $200 \mathrm{~nm}$ of $\mathrm{Au}$. The time between the two sputters was made less than 30 sec. in order to prevent the oxidation of $\mathrm{Cr}$ and consequently bad adhesion between $\mathrm{Cr}$ and $\mathrm{Au}$, which degrades the resistance of the $\mathrm{Cr} / \mathrm{Au}$ metallization mask to ammonium bifluoride $\left(\mathrm{NH}_{4} \mathrm{~F}_{2}\right)$. Photolithography was used for patterning the masking structure. The Au and $\mathrm{Cr}$ layers were wet etched at room temperature. Then the photoresist, Rohm and Haas S1813, was stripped using Rohm and Haas 1165 remover. The previous procedure was repeated on the back side of the wafer. Appropriate alignment marks were added to the mask in order to help in the alignment of the front side structure to that on the back side using the Neutronix Quintel NXQ4004 contact mask aligner. After patterning the masking structure, the tuning fork structures were etched in a solution of $60 \% \mathrm{NH}_{4} \mathrm{~F}_{2}$ in water heated at $80{ }^{\circ} \mathrm{C}$, to give approximately $40 \mu \mathrm{m} /$ hour etching rate in the $z$-axis direction of the quartz crystal. Although it took approximately 5 hours to fully etch the $350 \mu \mathrm{m}$ thick quartz wafer, the samples were left in the etching bath for 3 additional hours to smoothen the side surfaces of the tuning fork structure. Figure 3 shows one tuning fork structure after etching in $\mathrm{NH}_{4} \mathrm{~F}_{2}$. The $\mathrm{Au}$ and $\mathrm{Cr}$ layers were then removed using $\mathrm{Au}$ and $\mathrm{Cr}$ etchants.

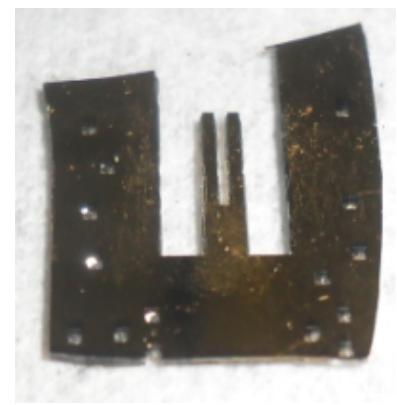

Fig. 3. Photograph of a tuning fork structure after etching in $\mathrm{NH}_{4} \mathrm{~F}_{2}$.

A major challenge in this work was the metallization of side electrodes. This had to be done through a stencil mask and not by conventional photolithography. We have elected a flexible polymide substrate to be used as a stencil mask material and this is due its availability and ease of processing. After forming the tuning fork structure, the electrodes were shadow sputtered through the stencil mask. The stencil mask was laser micromachined using LPKF Protolaser U laser prototyping machine. The machining was made using a $355 \mathrm{~nm}$ laser diode with an output power of 6.8 Watts at a frequency of $40 \mathrm{kHz}$. Finally, Aluminum (Al) was shadow sputtered through a stencil mask in order to form the top and side electrodes on one tine. A schematic diagram for the implemented drive and sense electrode pattern structure on the top face, bottom face, outer side, and inner side is shown in Figs. $4 \mathrm{a}-\mathrm{d}$. The sense electrodes were placed at the end of the tines while the drive electrodes were placed near the stem. The placement is such that the sense electrodes are placed in the most vibrationally active area. The gyroscope sensor was mounted on a ceramic chip carrier and then wire bonded to an external circuit. (a)

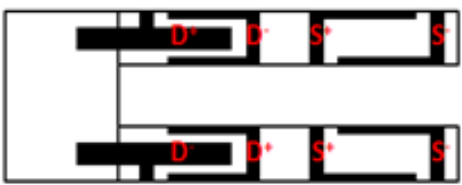

(b)

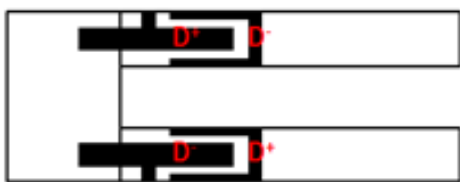

(c)

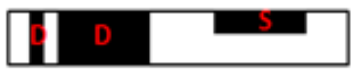

(d)

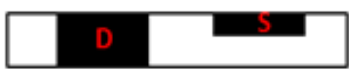

Top Face

\section{Bottom Face}

Outer Side

Inner Side
Fig. 4. Two-dimensional schematic diagram for the electrode pattern structure: a) top face, b) bottom face, c) outer side, and d) inner side.

\section{Measurements and discussion}

The gyroscope mounted on the electronic circuit board, was placed on Ideal Aerosmith's 1270VS z-axis rotation rate table, Fig. 5 . The rate table was rotated at different rates varying from $0 \% / \mathrm{s}$ to $60 \% / \mathrm{s}$ in steps of $20 \% / \mathrm{s}$ in both clockwise and anti-clockwise directions. The sensed signals were amplified using charge sensitive amplifiers. The signals were then fed to a differential amplifier in order to further amplify the signals and to cancel the common mode capacitive coupling present on the sense electrodes. The input differential amplification stage of Amptek's 5210 lock-in amplifier was used as the differential amplifier in our setup. The differentially amplified signal was then filtered and fed to Agilent's 35670A signal analyzer, from which the signal spectrum was captured using Labview. 


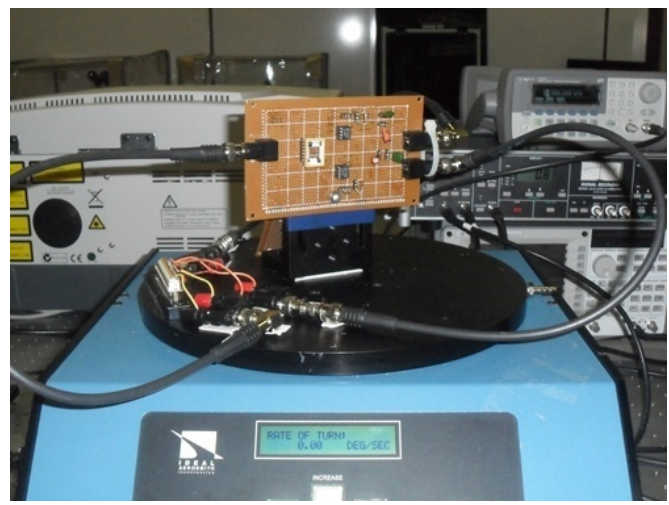

Fig. 5. A photograph of the gyroscope test setup.

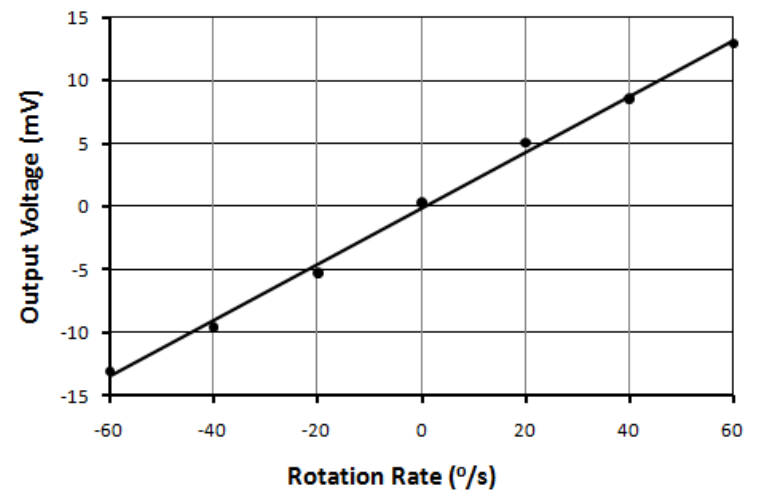

Fig. 6. Output signal versus input angular rate for the fabricated tuning fork quartz gyroscope.

The detected voltage versus the input angular rotation rate was recorded as shown in Fig. 6. The scale factor (sensitivity) of the gyroscope sensor is the slope of the detected voltage versus input angular rate plot. The gyroscope sensitivity is measured to be $0.22 \mathrm{mV} /{ }^{\circ} / \mathrm{s}$. The maximum rotational rate is $60 \%$ sec at a resonant frequency of $26324.25 \mathrm{~Hz}$ after which the detected signal saturates and the gyroscope becomes insensitive. It was noticed that the frequency varied slightly at different rotational rates. Figure 7 shows the gyroscope detected signal spectrum measured at a rotational rate of $60 \% / \mathrm{s}$ using the signal analyzer. The signal to noise ratio was found to be approximately 40 .

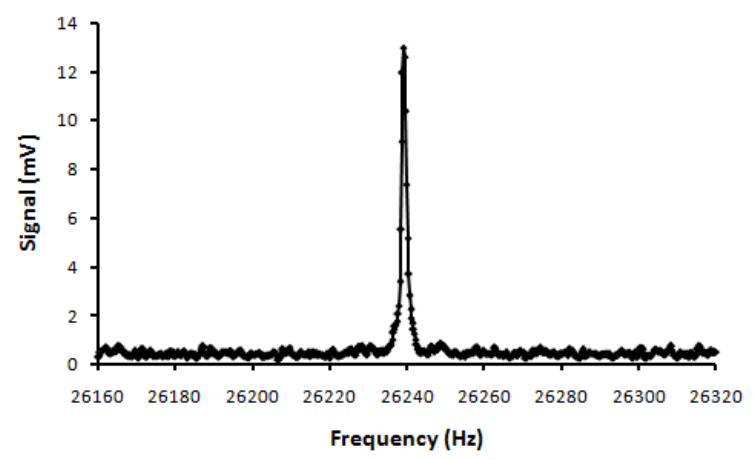

Fig. 7. Detected spectrum for gyroscope at a rotation rate of $60 \%$ s.

\section{Conclusions}

In this paper, we reported an observed driveless response of a quartz gyroscope with sensitivity and signal to noise ratio of $0.22 \mathrm{mV} / \% / \mathrm{s}$ and 40 , respectively, at a rotational rate of $60^{\circ} / \mathrm{s}$. The measured response signal can be increased by adding electrodes and sensing the signals from both the top and bottom faces of both tines. The observed driveless operation eliminates the need for an oscillator drive circuitry.

\section{Acknowledgments}

This work was supported by NSTIP strategic technologies program number 09-ELE665-02 in the Kingdom of Saudi Arabia. In addition, the authors extend their appreciation to the Deanship of Scientific Research at King Saud University for funding this work through the Research Project no NFG2-11-33.

\section{References}

[1] A.M. Madni, L.A. Wan, S. Hammons, Proceedings of the Aerospace Applications Conference, IEEE, Aspen, CO, 2, 315 (1996).

[2] A.M. Madni, L.E. Costlow, S.J. Knowles, IEEE Sensors Journal 3(5), 569 (2003).

[3] S.D. Senturia, Microsystem Design, Kluwer Academic Publishers, Norwell, MA, 2001.

[4] J. Soderkvist, Proceedings of the 44th Annual Symposium on Frequency Control, Baltimore, MD, p. 406 1990. 